\title{
Editorial \\ Electromagnetic Scattering and Its Applications: From Low Frequencies to Photonics
}

\author{
Alessandro Fedeli *(D) and Gian Luigi Gragnani *(D) \\ Department of Electrical, Electronic, Telecommunications Engineering, and Naval Architecture, \\ University of Genoa, Via all'Opera Pia, 11A, 16145 Genoa, Italy \\ * Correspondence: alessandro.fedeli@unige.it (A.F.); gianluigi.gragnani@unige.it (G.L.G.)
}

check for updates

Citation: Fedeli, A.; Gragnani, G.L. Electromagnetic Scattering and Its Applications: From Low Frequencies to Photonics. Electronics 2021, 10, 2352. https://doi.org/10.3390/ electronics10192352

Received: 22 September 2021 Accepted: 24 September 2021 Published: 26 September 2021

Publisher's Note: MDPI stays neutral with regard to jurisdictional claims in published maps and institutional affiliations.

Copyright: (c) 2021 by the authors. Licensee MDPI, Basel, Switzerland. This article is an open access article distributed under the terms and conditions of the Creative Commons Attribution (CC BY) license (https:// creativecommons.org/licenses/by/ $4.0 /)$.
Few research topics are as broad and pervasive as electromagnetic scattering. Undeniably, electromagnetic scattering phenomena are behind many wireless and radio devices. From radar systems to mobile telecommunications, and from medical devices to innovative materials, the study of electromagnetic interactions and scattering is fundamental to develop applications that exploit electromagnetic waves.

Although the initial research in the field dates back centuries, many unresolved theoretical issues are still there. At the same time, novel applications of electromagnetic scattering are continuously emerging. A detailed description of the many areas in which scattering is involved is well beyond our scope. Based on the wavelength, applications may be very different, as well as the techniques used to analyze and simulate the electromagnetic propagation.

Starting from low-frequency problems and embracing the whole spectrum up to optics and photonics, the goal of the present Special Issue is to provide a comprehensive collection of state-of-the-art papers dedicated to electromagnetic scattering theory and applications.

Electromagnetic scattering from the so-called canonical structures is one of the subjects covered in this Special Issue. In particular, a theoretical framework is presented in [1] to provide a description of scattering phenomena involving dielectric and conducting spheres by decomposing the total field in the outer space in terms of inward and outward electromagnetic fields, rather than in terms of incident and scattered fields, as in the classical Lorenz-Mie formulation. The method can provide an intuitive physical interpretation of electromagnetic scattering in terms of impedance matching and resonances.

Of course, when the structures under consideration become more complex, the development of proper computational techniques is necessary to simulate and analyze the electromagnetic scattering effects. An example is the study of scattering problems in the presence of bianisotropic materials and metamaterials. In this regard, reference solutions obtained by means of finite-elements approximations are presented in [2]. In this paper, not only the well-posedness and the finite-element approximability in the three-dimensional and time-harmonic case are discussed, but also three example problems are considered: one, in free space, deals with scattering from plasmonic gratings that exhibit bianisotropy, while the other two deal with bianisotropic obstacles inside waveguides.

The propagation of electromagnetic waves in magnetized plasma is another research topic that deserves particular attention. In this Special Issue, this problem is addressed in [3], where a numerical method based on a modified precise-integration time-domain formulation is illustrated. It is shown that the method can provide an evident reduction in the execution time by using a larger simulated time step; meanwhile, the computational error of the presented algorithm is also lower than those of the formulations based on the FDTD scheme, especially in the high-frequency range.

In some applications it is important to study the scattering effects (e.g., the radar cross section) of large structures with details of different scales. In this situation, the adoption of full-wave numerical methods for the whole targets under test may require very high computational resources. A possible solution is represented by the use of hybrid techniques, 
in which full-wave methods may be combined with approaches based on high-frequency approximations. This is the topic of the work presented in [4], where the finite volume time domain technique is hybridized with an asymptotic solution strategy based on physical optics and the equivalent current method.

Electromagnetic band gap structures (EBGs) [5], despite having been introduced more than twenty years ago [6], still continue to have a significant impact in microwave and antenna engineering research. This topic is considered in [7], where three different types of microstrip band stop filters based on EBGs are proposed and compared, with exagonal, octagonal, and elliptical rings.

Mobile telecommunications are another field where scattering phenomena are unavoidable and strongly contribute to the resulting electromagnetic field levels and possible coverage issues. This point may be critical if irregular geometries are involved. In [8], a model for the estimation of electromagnetic field levels in built-up areas, which enhances the COST231-Walfisch-Ikegami model $[9,10]$, and able to effectively deal with the built-up scenarios of hilly, largely variable, and small, irregularly arranged towns, is developed and validated from an experimental viewpoint.

Finally, another interesting topic covered in this Special Issue is inverse electromagnetic scattering, with reference to the problem of microwave imaging. In particular, tomographic microwave imaging of dielectric targets inside circular conducting cylinders is discussed in [11], analyzing the performance of a nonlinear quantitative inversion technique in non-Hilbertian Lebesgue spaces. The approach studied by the authors could be exploited in many applications making use of circular enclosures, such as the case of biomedical imaging where the process requires a tight coupling of the investigated region with the surrounding background media.

Funding: This research received no external funding.

Conflicts of Interest: The authors declare no conflict of interest.

\section{References}

1. Ruello, G.; Lattanzi, R. Scattering from Spheres: A New Look into an Old Problem. Electronics 2021, 10, 216, [CrossRef]

2. Kalarickel Ramakrishnan, P.; Raffetto, M. Three-Dimensional Time-Harmonic Electromagnetic Scattering Problems from Bianisotropic Materials and Metamaterials: Reference Solutions Provided by Converging Finite Element Approximations. Electronics 2020, 9, 1065. [CrossRef]

3. Kang, Z.; Huang, M.; Li, W.; Wang, Y.; Yang, F. An Efficient Numerical Formulation for Wave Propagation in Magnetized Plasma Using PITD Method. Electronics 2020, 9, 1575. [CrossRef]

4. Fedeli, A.; Pastorino, M.; Randazzo, A. A Hybrid Asymptotic-FVTD Method for the Estimation of the Radar Cross Section of 3D Structures. Electronics 2019, 8, 1388. [CrossRef]

5. Rahmat-Samii, Y.; Mosallaei, H. Electromagnetic band-gap structures: Classification, characterization, and applications. In Proceedings of the 2001 Eleventh International Conference on Antennas and Propagation, Manchester, UK, 17-20 April 2001; Volume 2, pp. 560-564. [CrossRef]

6. Sievenpiper, D.F. High-impedance electromagnetic surfaces. Ph.D. Thesis, University of California, Los Angeles, CA, USA, 1999.

7. Zheng, X.; Jiang, T.; Lu, H.; Wang, Y. Double-Layer Microstrip Band Stop Filters Etching Periodic Ring Electromagnetic Band Gap Structures. Electronics 2020, 9, 1216. [CrossRef]

8. Schirru, L.; Ledda, F.; Lodi, M.B.; Fanti, A.; Mannaro, K.; Ortu, M.; Mazzarella, G. Electromagnetic Field Levels in Built-up Areas with an Irregular Grid of Buildings: Modeling and Integrated Software. Electronics 2020, 9, 765. [CrossRef]

9. Ikegami, F.; Yoshida, S.; Takeuchi, T.; Umehira, M. Propagation factors controlling mean field strength on urban streets. IEEE Trans. Antennas Propag. 1984, 32, 822-829. [CrossRef]

10. Walfisch, J.; Bertoni, H. A theoretical model of UHF propagation in urban environments. IEEE Trans. Antennas Propag. 1988, 36, 1788-1796. [CrossRef]

11. Fedeli, A.; Pastorino, M.; Randazzo, A.; Gragnani, G.L. Analysis of a Nonlinear Technique for Microwave Imaging of Targets Inside Conducting Cylinders. Electronics 2021, 10, 594. [CrossRef] 